\title{
Symbolverzeichnis Band II
}

\author{
(alphabetisch)
}

\begin{tabular}{|c|c|}
\hline$A$ & Amplitude \\
\hline$\hat{\bar{a}}$ & Viererbeschleunigung \\
\hline$B$ & Beweglichkeit \\
\hline$b$ & Stoßparameter \\
\hline C & Wärmekapazität \\
\hline$C_{P}^{m}, C_{V}^{m}$ & Molwärme bei konstantem Druck und konstantem Volumen \\
\hline$c$ & Lichtgeschwindigkeit im Vakuum (c = 299792458 m/s) \\
\hline$c, c_{P}, c_{V}$ & spezifische Wärme (bei konstantem Druck oder konstantem Volumen) \\
\hline$D$ & Diffusionskoeffizient \\
\hline$d$ & Durchmesser \\
\hline$d_{F}$ & Hausdorff-Dimension \\
\hline$E, E_{\mathrm{kin}}, E_{\mathrm{pot}}$ & Energie, Gesamtenergie (relativistisch), kinetische und potenzielle Energie \\
\hline$E_{0}=m c^{2}$ & Ruheenergie (rest energy) \\
\hline$F$ & freie Energie, Kraft, Faraday-Konstante \\
\hline$\hat{\bar{F}}$ & Viererkraft, Minkowski-Kraft \\
\hline$f(v), n(v)$ & Verteilungsfunktion (Geschwindigkeitsbetrag) \\
\hline$f\left(E_{\mathrm{kin}}\right)$ & Verteilungsfunktion (Energie) \\
\hline$f(\vec{v}), F(\vec{v})$ & Verteilungsfunktion (Geschwindigkeit) \\
\hline$f, v$ & Zahl der Freiheitsgrade \\
\hline GG & Gleichgewicht \\
\hline$G, g$ & freie Enthalpie, freie Enthalpie pro Mol \\
\hline$H$ & Enthalpie \\
\hline I & elektrische Stromstärke, Trägheitsmoment \\
\hline$j$ & Teilchenstromdichte \\
\hline$k$ & Federkonstante (Oszillator) \\
\hline$k$ & Boltzmannkonstante \\
\hline$l_{0}$ & Eigenlänge (proper length) \\
\hline$L_{T}, L_{T}^{V}$ & Umwandlungswärme, Verdampfungswärme \\
\hline$M_{m}$ & Molmasse \\
\hline$m_{M}$ & absolute Molekülmasse (früher Molekulargewicht) \\
\hline$M_{R}$ & relative Molkülmasse \\
\hline MP & Massenpunkt, Teilchen \\
\hline$N$ & Teilchenzahl \\
\hline$N_{A}$ & Avogadrozahl \\
\hline$n=N / V$ & Teilchendichte \\
\hline$n(p)$ & Verteilungsfunktion (Impuls) \\
\hline$P$ & Druck, Wahrscheinlichkeit \\
\hline$p$ & Impuls \\
\hline$\vec{p}_{R}=m \gamma \vec{v}$ & relativistischer Impuls (Raumanteil) \\
\hline$\hat{\bar{p}}$ & Viererimpuls \\
\hline$Q$ & Wärmemenge, elektrische Energie \\
\hline$\dot{Q}, \dot{q}$ & Wärmestrom, Wärmestromdichte \\
\hline$R$ & Gaskonstante \\
\hline $\mathrm{Ra}$ & Rayleigh-Zahl \\
\hline $\mathrm{R}_{\mathrm{S}}$ & spezifische Gaskonstante \\
\hline$R T$ & Raumtemperatur $\left(20^{\circ} \mathrm{C}\right)$ \\
\hline
\end{tabular}

https://doi.org/10.1515/9783110675696-208 


\section{$\hat{\vec{r}} \quad$ Ereignisvektor}

$S, S^{\prime} \quad$ Licht-Kugelwelle

$S, s, S_{m}$

Entropie, spezifische Entropie $\left(s=\frac{S}{m}\right)$, molare Entropie $\left(S_{m}=\frac{S}{v}\right)$

$s^{2}, d s^{2}, d \hat{\vec{s}}^{2}$

Abstandsquadrat, Ereignisintervall

$T$

absolute Temperatur in Kelvin

$T_{3}$

Tripelpunkt, $T_{3}=273,16 \mathrm{~K}$ (exakt)

$U$

$u=U / V$ innere Energie, elektrische Spannung

V

spezifische innere Energie

$V_{m}$

Volumen

molares Volumen

Geschwindigkeit

$\hat{\vec{v}}$

W

Vierergeschwindigkeit

WW

Arbeit, Wasserwert

$\vec{X}(t)$

Wechselwirkung

$x_{f}$

Zustandsvektor (nichtlineare Dynamik)

$Z$

Fixpunkt (nichtlineare Dynamik)

kanonische Zustandssumme

$\alpha$

Ausdehnungskoeffizient (Gase, Feststoffe)

$\beta$

Spannungskoeffizient, Geschwindigkeitsparameter $\left(\beta=\frac{v}{c}\right)$

Volumsausdehnungskoeffizient (Flüssigkeiten), Dämpfungskonstante,

$$
\text { Lorentz-Faktor }\left(\gamma=\frac{1}{\sqrt{1-\beta^{2}}}\right)
$$

Bifurkationsabstand

$\delta \quad$ Feigenbaum-Konstante

$\varepsilon \quad$ Leistungszahl

$\eta$

$\theta$

$\vartheta$

$\kappa$

Wirkungsgrad, Zähigkeit

Sichtwinkel

Temperatur in Grad Celsius Adiabatenkoeffizient
(Selbstähnlichkeit) $\left(\kappa=\frac{c_{P}}{c_{V}}\right)$, Kompressionsmodul, Skalenexponent

Wärmeleitfähigkeit, Wärmeleitzahl

$\lambda$

mittlere freie Weglänge, Lyapunov-Exponent, Skalenfaktor (Selbstähnlichkeit),

Wellenlänge

$\mu \quad$ magnetisches Moment

$\mu_{J T}$

Joule-Thomson Koeffizient

$v$

Stoffmenge, Molzahl, Stoßfrequenz, Frequenz von Lichtquellen

Korrelationslänge

$\rho$

Dichte, spezifischer elektrischer Widerstand

$\Sigma, \Sigma^{\prime}$

gegeneinander bewegte Systeme

$\sigma$

Streuquerschnitt, Kontrollparameter (nichtlineare Dynamik)

$\sigma_{\infty} \quad$ Feigenbaum-Punkt

$\tau$

mittlere Stoßzeit, Relaxationszeit

$\Delta \tau$

Eigenzeitintervall (proper time interval)

Pendelauslenkung 
$\Omega$ $\omega, \omega_{0}$
Zahl der zugänglichen Mikrozustände (mikrokanonische Zustandssumme)

Frequenz (Kreisfrequenz), Eigenfrequenz (Kreisfrequenz)

Wichtige physikalische Größen, Band II

Lichtgeschwindigkeit

Ausdehnungskoeffizient

idealer Gase bei Normbedingungen

$\left(P=1 \mathrm{~atm}=1,01325 \mathrm{~Pa}, T=0{ }^{\circ} \mathrm{C}=273,15 \mathrm{~K}\right)$

Molvolumen unter Normbedingungen

absolute Temperatur

Universelle Gaskonstante

Avogadro-Konstante

Boltzmannkonstante

Kalorie

Feigenbaum-Konstante

atomare Masseneinheit (atomic mass unit) $c=299792458 \mathrm{~m} / \mathrm{s}$

$\alpha_{T_{3}}=\frac{1}{273,15 K}=\frac{1}{T_{3}}$

$V_{m, S T P}=22,414 \cdot 10^{-3} \mathrm{~m}^{3} / \mathrm{mol}=22,414(\mathrm{dm})^{3} \mathrm{~mol}^{-1}$

$1 \mathrm{~K}=\left(1,380649 \cdot 10^{-23} / \mathrm{k}\right) \mathrm{J}$,

k... Boltzmannkonstante

$R=k \cdot N_{A}=8,314462618 \ldots \mathrm{J} \cdot \mathrm{mol}^{-1} \mathrm{~K}^{-1}$

$N_{A}=6,02214076 \cdot 10^{23} \mathrm{~mol}^{-1}$

$k=1,380649 \cdot 10^{23} \mathrm{~J} \cdot \mathrm{K}^{-1}$

$1 \mathrm{cal}=4,186 \mathrm{~J}$

$\delta=4,66920160910 \ldots$

$1 \mathrm{u}=1,6605 \cdot 10^{-27} \mathrm{~kg}$ 
